\title{
Acute and long-term disruption of glycometabolic control after SARS-CoV-2 infection
}

\author{
Laura Montefusco ${ }^{1,16}$, Moufida Ben Nasr ${ }^{2,3,16}$, Francesca D'Addio ${ }^{2,16}$, Cristian Loretelli ${ }^{2}{ }^{2}$, \\ Antonio Rossi', Ida Pastore ${ }^{1}$, Giuseppe Daniele ${ }^{4}$, Ahmed Abdelsalam ${ }^{\circ}{ }^{2}$, Anna Maestroni², \\ Marco Dell'Acqua ${ }^{2,5}$, Elio Ippolito ${ }^{2}{ }^{2}$, Emma Assi ${ }^{2}{ }^{2}$, Vera Usuelli ${ }^{2}$, Andy Joe Seelam ${ }^{2}{ }^{2}$, \\ Roberta Maria Fiorina ${ }^{2}$, Enrica Chebat', Paola Morpurgo', Maria Elena Lunati', \\ Andrea Mario Bolla', Giovanna Finzi ${ }^{6}$, Reza Abdi ${ }^{7}$, Joseph V. Bonventre ${ }^{7}{ }^{7}$, Stefano Rusconi ${ }^{8}{ }^{8}$, \\ Agostino Riva $\oplus^{8}$, Domenico Corradi $\odot^{9}$, Pierachille Santus ${ }^{10,11}$, Manuela Nebuloni ${ }^{12,13}$, Franco Folli ${ }^{14}$, \\ Gian Vincenzo Zuccotti ${ }^{1,15}$, Massimo Galli ${ }^{8}$ and Paolo Fiorina ${ }^{1,2,3 凶}$
}

\begin{abstract}
Patients with coronavirus disease 2019 (COVID-19) are reported to have a greater prevalence of hyperglycaemia. Cytokine release as a consequence of severe acute respiratory syndrome coronavirus 2 infection may precipitate the onset of metabolic alterations by affecting glucose homeostasis. Here we describe abnormalities in glycometabolic control, insulin resistance and beta cell function in patients with COVID-19 without any pre-existing history or diagnosis of diabetes, and document glycaemic abnormalities in recovered patients 2 months after onset of disease. In a cohort of 551 patients hospitalized for COVID-19 in Italy, we found that $46 \%$ of patients were hyperglycaemic, whereas $27 \%$ were normoglycaemic. Using clinical assays and continuous glucose monitoring in a subset of patients, we detected altered glycometabolic control, with insulin resistance and an abnormal cytokine profile, even in normoglycaemic patients. Glycaemic abnormalities can be detected for at least 2 months in patients who recovered from COVID-19. Our data demonstrate that COVID-19 is associated with aberrant glycometabolic control, which can persist even after recovery, suggesting that further investigation of metabolic abnormalities in the context of long COVID is warranted.
\end{abstract}

D iabetes is one of the more frequent comorbidities of severe acute respiratory syndrome coronavirus 2 (SARS-CoV-2), which can cause lethal respiratory disease ${ }^{1-4}$. Preliminary studies reported greater prevalence of hyperglycaemia in a cohort of patients affected by COVID-19 (refs. 5,6 ); similar data were previously reported in patients affected by SARS-CoV-1, which has been shown to increase the levels of fasting glucose as compared to glucose levels observed in patients with pneumonia unrelated to SARS-CoV-1 infection ${ }^{7-9}$. Currently, little evidence exists as to whether the effect of SARS-CoV-2 on beta cell function is direct or indirect ${ }^{10,11}$. It is theoretically possible that SARS-CoV-2 may localize to the endocrine pancreas; indeed, mRNA levels of angiotensin-converting enzyme 2 (ACE2), which is the primary SARS-CoV-2 receptor, were found to be high in both exocrine and endocrine pancreas ${ }^{8,12}$, and immunohistochemistry as well as in situ hybridization studies have identified SARS-CoV-1-related antigen in the pancreases of patients who died of SARS-CoV-1 (refs. ${ }^{13,14}$ ). Recently, the presence of SARS-CoV-2 antigen in the post-mortem pancreases of patients who died of COVID-19 has been reported as well ${ }^{15}$. Moreover, studies using primary human islets demonstrated that pancreatic beta cells are highly permissive to SARS-CoV-2 infection through ACE2 (refs. ${ }^{16,17}$ ). Interestingly, SARS-CoV-2 also induces a cytokine storm, an exaggerated immune response with a broad spectrum of cytokine production that establishes a systemic proinflammatory milieu, which may play a role in facilitating insulin resistance and beta cell hyperstimulation, eventually leading to altered beta cell function and death ${ }^{16,18-20}$. SARS-CoV-2 may also enhance the pre-existing proinflammatory status observed in type 2 diabetes $^{21-24}$ (T2D), thus worsening patient survival and complications. The aim of this study is to examine whether abnormalities in glycometabolic control, insulin resistance and beta cell function are associated with COVID19 in patients ('acute COVID-19') without any pre-existing history or diagnosis of diabetes. We also evaluated the persistence of these abnormalities over time in patients who recovered from COVID-19 ('post COVID-19'). Our study demonstrates the manner in which

\footnotetext{
'Division of Endocrinology, ASST Fatebenefratelli-Sacco, Milan, Italy. ${ }^{2}$ International Center for T1D, Pediatric Clinical Research Center Romeo ed Enrica Invernizzi, DIBIC, Università di Milano, Milan, Italy. ${ }^{3}$ Nephrology Division, Boston Children's Hospital, Harvard Medical School, Boston, MA, USA. ${ }^{4}$ Metabolic Diseases, Department of Medicine, University of Pisa, Pisa, Italy. ${ }^{5}$ Division of Endocrinology, Aziende Socio Sanitarie Territoriali Fatebenefratelli Sacco, Milan, Italy. ${ }^{6}$ Department of Pathology, University Hospital ASST-Settelaghi, Varese, Italy. ${ }^{7}$ Renal Division and Transplantation Research Center, Brigham and Women's Hospital, Harvard Medical School, Boston, MA, USA. ${ }^{8}$ Infectious Diseases Unit, ASST Fatebenefratelli-Sacco, Milan, Italy. 'Department of Biomedical, Biotechnological and Translational Sciences, Unit of Pathology, University of Parma, Parma, Italy. ${ }^{10}$ Division of Respiratory Diseases, Ospedale L. Sacco, ASST Fatebenefratelli-Sacco, Milan, Italy. "Department of Biomedical and Clinical Sciences, DIBIC, Università di Milano, Milan, Italy. ${ }^{12}$ Department of Pathology, Papa Giovanni XXIII Hospital, Bergamo, Italy. ${ }^{13}$ Department of Biomedical and Clinical Sciences, Università di Milano, Milan, Italy. ${ }^{14}$ Endocrinology and Metabolism, Department of Health Science, Università di Milano, ASST Santi Paolo e Carlo, Milan, Italy. ${ }^{15}$ Department of Pediatrics, Children's Hospital Buzzi, Università di Milano, Milan, Italy. ${ }^{16}$ These authors contributed equally: Laura Montefusco, Moufida Ben Nasr, Francesca D’Addio. $\bowtie$ e-mail: paolo.fiorina@childrens.harvard.edu
} 
COVID-19-related hyperglycaemia develops and thus may aid in determining the mechanism of disease.

\section{Results}

Increased rate of new-onset hyperglycaemia in COVID-19. We first evaluated alterations in glycometabolic control in a cohort of 551 patients with COVID-19 admitted to our academic centre (ASST FBF-Sacco Milan, Presidio Sacco). A total of 151 patients (27\%) were affected by T2D with clearly abnormal levels of glycated haemoglobin at hospital admission (Fig. 1a,c). Among these 151 patients, 86 had a history of diabetes, while a diabetes diagnosis was made for the remaining 65 patients according to the American Diabetes Association (ADA) criteria $^{25}$ during their in-hospital stay (Fig. 1a). Surprisingly, in 253 of 551 patients (46\%), overt hyperglycaemia was measured during hospitalization, while the remaining 147 patients (27\%) displayed normal blood glucose levels (Fig. 1a). Among patients who exhibited new-onset hyperglycaemia at hospital admission for COVID-19, persistent hyperglycaemia continued to be observed in the following 6 months in nearly $35 \%$ of patients, overt diabetes was diagnosed in $\sim 2 \%$ of patients, and the remaining $63 \%$ of patients showed remission and became normoglycaemic (Fig. 1b). Demographic and clinical characteristics of the study population are reported in Table 1. Importantly, the mean time to clinical improvement from COVID-19 was $14.9 \pm 0.5 \mathrm{~d}$ for all patients, although it was extended to $20.2 \pm 1.3 \mathrm{~d}$ in the previously diabetic group (Table 1). As expected, mean haemoglobin A1c (HbA1c) levels were significantly higher in patients with established/newly diagnosed diabetes as compared to patients with new-onset hyperglycaemia and normoglycaemia, and HbA1c levels did not differ between patients with normoglycaemia and patients with new-onset hyperglycaemia (Fig. 1c), thus confirming the recent onset of hyperglycaemia. Conversely, mean peak blood glucose levels measured during the hospital stay were significantly different among the three groups, and blood glucose levels of patients who had established diabetes were the highest (Fig. 1d). A time-to-event endpoint analysis showed increased mortality in patients with established/newly diagnosed diabetes, with an increase in the hazard ratio (HR) as compared to patients with normoglycaemia (HR: 2.16, confidence interval $(\mathrm{CI}): 1.27-3.67, P=0.009)$ and those with new-onset hyperglycaemia (HR: 2.05, CI: 1.28-3.29, $P=0.002$; Fig. 1e). Interestingly, patients with new-onset hyperglycaemia required a longer in-hospital stay (Fig. 1f) and displayed a higher clinical score at hospital admission (Fig. 1g), which was also associated with a higher proportion of patients who had new-onset hyperglycaemia requiring oxygen support and ventilation as compared to patients with normoglycaemia, while no difference in the need for intensive care was reported among groups (Fig. $1 \mathrm{~h}-\mathrm{j}$ ). An increased odds ratio observed in hyperglycaemic patients, after adjusting for age and sex (Fig. 1k), further confirmed the association between new-onset hyperglycaemia and poor clinical outcomes. Additionally, patients with established T2D required a longer hospitalization stay and displayed worse clinical scores and respiratory parameters as compared to the two other groups (Fig. 1f-j). Taken together, our data suggest that COVID-19-associated new-onset hyperglycaemia may predispose patients to long-term hyperglycaemia, worse clinical outcomes and clinical scores, prolonged hospital stays and higher demand for oxygen support or positive-pressure ventilation.

\section{Continuous glucose monitoring demonstrated glycaemic abnor-} malities in COVID-19. To evaluate alterations in glycaemic control not detected by fasting glycaemia, professional continuous glucose monitoring (CGM) was performed in patients with COVID-19 $(n=10)$ or in patients who eventually recovered from COVID-19 $(n=10)$ and with normal fasting glucose, in healthy controls $(n=15)$ and in patients with T2D ( $n=10$; Fig. $2 \mathrm{a}-\mathrm{h})$. CGM was performed during the acute phase of COVID-19 and at $62.0 \pm 6.5 \mathrm{~d}$ after disease onset (Table 2 and Supplementary Table 1). Demographic and clinical characteristics of the aforementioned subgroups are reported in Table 2. Analysis of CGM reports collected from subgroups showed that, in normoglycaemic patients, COVID-19 was associated with an overall impaired glycaemic profile, as demonstrated by a significantly longer duration of glycaemia above $140 \mathrm{mg}$ $\mathrm{dl}^{-1}$ (Fig. 2a), significantly higher glycaemic area under the curve (AUC) above $140 \mathrm{mg} \mathrm{dl}^{-1}$ (Fig. 2b) and higher mean postprandial glycaemia at $60 \mathrm{~min}$ (Fig. 2c). COVID-19 was also associated with higher glycaemic variability, as shown by higher coefficient of variability (Fig. 2e) and higher standard deviation (Fig. 2f) as compared to healthy controls. Surprisingly, glycaemic alterations persisted in some patients who recovered from COVID-19. Indeed, compared to healthy controls, patients who recovered from COVID-19 showed a greater duration of glycaemia above $140 \mathrm{mg} \mathrm{dl}^{-1}$ (Fig. 2a), higher mean postprandial glycaemia at $120 \mathrm{~min}$ (Fig. 2d), higher mean blood glucose (Fig. $2 \mathrm{~g}$ ) and higher nadir blood glucose (Fig. $2 \mathrm{~h})$. For patients who recovered from COVID-19, other parameters such as coefficient of variability and standard deviation were similar to those of healthy controls but different from those of patients with acute COVID-19 (Fig. 2e,f). Collectively, our findings suggest that abnormal glycometabolic control occurs in patients with COVID-

Fig. 1 | Increased rate of new-onset hyperglycaemia in patients with COVID-19. a, Glycometabolic abnormalities in a cohort of 551 patients with COVID19 (acute COVID-19) at hospital admission. b, Glycaemic alterations for the hyperglycaemic group at 6 months follow-up from their hospital discharge (post COVID-19). c,d, Mean HbA1c levels and mean peak blood glucose levels were evaluated in patients with diabetes, new-onset hyperglycaemia and normoglycaemia. e, Survival rates of the three groups (diabetic, new-onset hyperglycaemic and normoglycaemic) represented as time to clinical endpoint analysis, showing an increase in mortality in the diabetic group as compared to the hyperglycaemic and normoglycaemic groups. $\mathbf{f}, \mathbf{g}$, Time to hospital discharge and clinical score at hospital admission in the three patient groups. $\mathbf{h}-\mathbf{j}$, Rate of oxygen requirement, ventilatory support and need for intensive care were also reported and compared in the diabetic, hyperglycaemic and normoglycaemic groups; dark grey rectangles represent individuals with diabetes, light grey rectangles represent individuals with hyperglycaemia, and white rectangles represent individuals with normoglycaemia. $\mathbf{k}$, Forest plots comparing the odds ratio of the clinical outcomes (oxygen support, ventilatory support and need for intensive care) between the hyperglycaemic and the normoglycaemic groups, after adjusting for age and sex. Bar plots in $\mathbf{a}$ and $\mathbf{b}$ represent the proportion of individuals with diabetes, hyperglycaemia and normoglycaemia. Scatterplots in $\mathbf{c}$ and $\mathbf{d}$ show the mean \pm s.e.m., the error bars represent the s.e.m., and each dot represents an individual sample (diabetic (black; $n=146$ ), hyperglycaemic (dark grey; $n=249$ ) and normoglycaemic (light grey; $n=140$ )). Survival curve in e represents the proportions of individuals at risk who are still alive at regular intervals, up to $30 \mathrm{~d}$ from admission and stratified by glycaemic status ((diabetic (grey lines), hyperglycaemic (blue lines) and normoglycaemic (green lines)). Bar graphs in $\mathbf{f}$ and $\mathbf{g}$ show the mean \pm s.e.m., and the error bars represent the s.e.m. (f: diabetic (black; $n=151$ ), hyperglycaemic (dark grey; $n=253$ ) and normoglycaemic (light grey; $n=147$ ) groups; $\mathbf{g}$ : diabetic (dark grey bars; $n=144$ ), hyperglycaemic (light grey bars; $n=247$ ) and normoglycaemic (white bars; $n=140$ ) groups. Stacked bar graphs in $\mathbf{h}$-j represent proportions of patients requiring or not requiring oxygen support (diabetic, $n=146$; hyperglycaemic, $n=221$; normoglycaemic, $n=126$ ), ventilatory support (diabetic, $n=146$; hyperglycaemic, $n=219$; normoglycaemic, $n=149$ ), intensive care need (diabetic, $n=143$; hyperglycaemic, $n=218$; normoglycaemic, $n=128$ ). Log-rank (Mantel-Cox) test (e), one-way analysis of variance (ANOVA) with Holm-Sidak correction (c, $\mathbf{d}$ and $\mathbf{f}$ ) or Kruskal-Wallis with Dunn's correction (g), two-sided Fisher's/ chi-squared test ( $\mathbf{h}, \mathbf{i}$ and $\mathbf{j})$ and logistic multivariable regression $(\mathbf{k})$ were used for statistical analysis. VS, ventilatory support; ICU, intensive care unit. 
19 , although to a lower extent as compared to glycaemic alterations observed in patients with T2D, and that this effect persists even after recovery from the disease.
Insulin resistance and beta cell hyperstimulation after COVID19. To evaluate the extent of insulin resistance and improper beta cell function, we performed serum hormone sampling under fasting

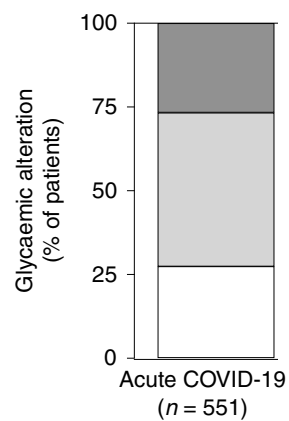

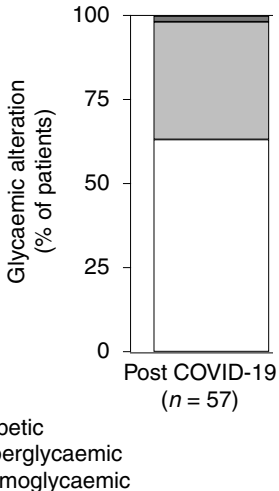

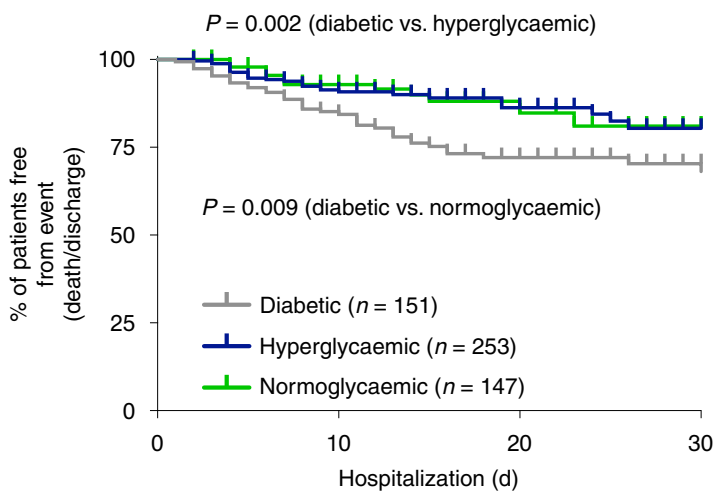

C

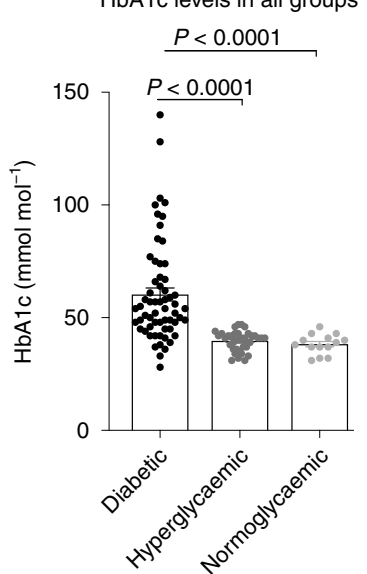

f

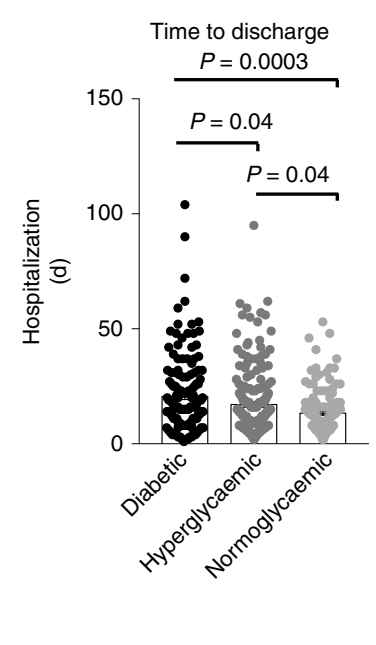

d Peak glycaemia values
during hospitalization

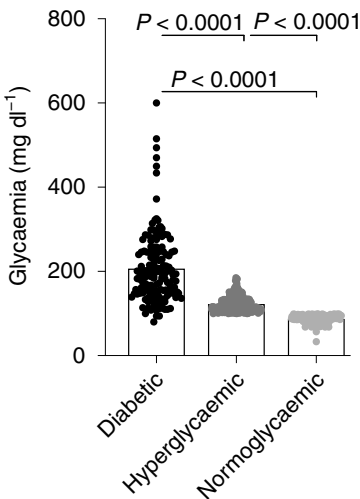

g

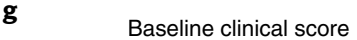

$\square$ Diabetic $(n=144)$

$\square$ Hyperglycaemic $(n=247)$

$\square$ Normoglycaemic $(n=140) \quad P<0.001$
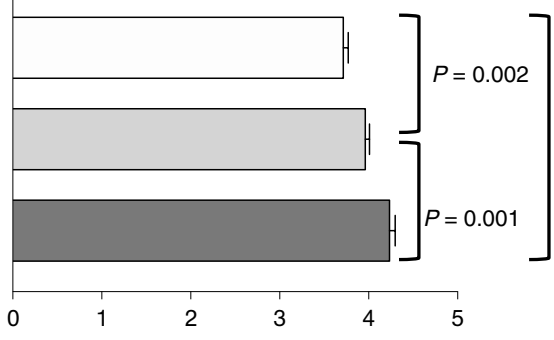

Baseline clinical score (1-6)
Number at risk

Diabetic

Hyperglycaemic

Normoglycaemic
151

253

147

$\begin{array}{rl}109 & 59 \\ 160 & 57 \\ 82 & 25\end{array}$

h $\square$ Diabetic
Hyperglycaemic
$\square$ Normoglycaemic

Oxygen support

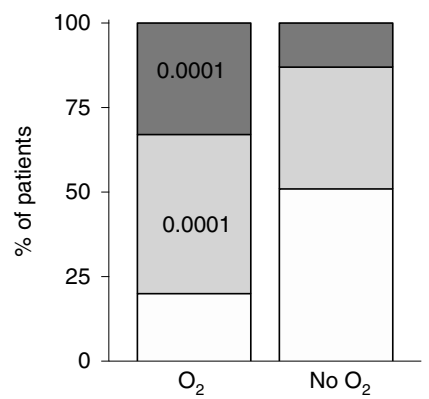

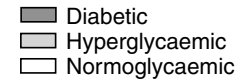

VS

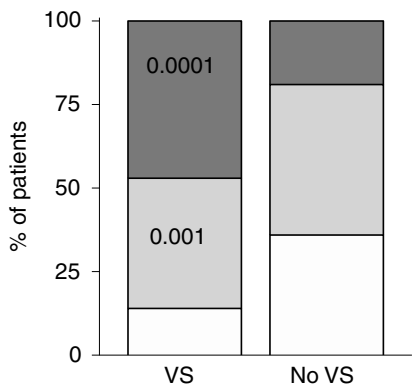

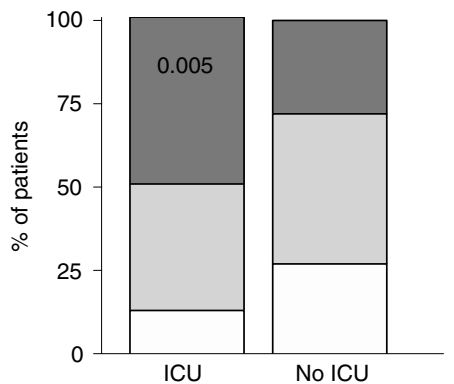

$\mathbf{k}$

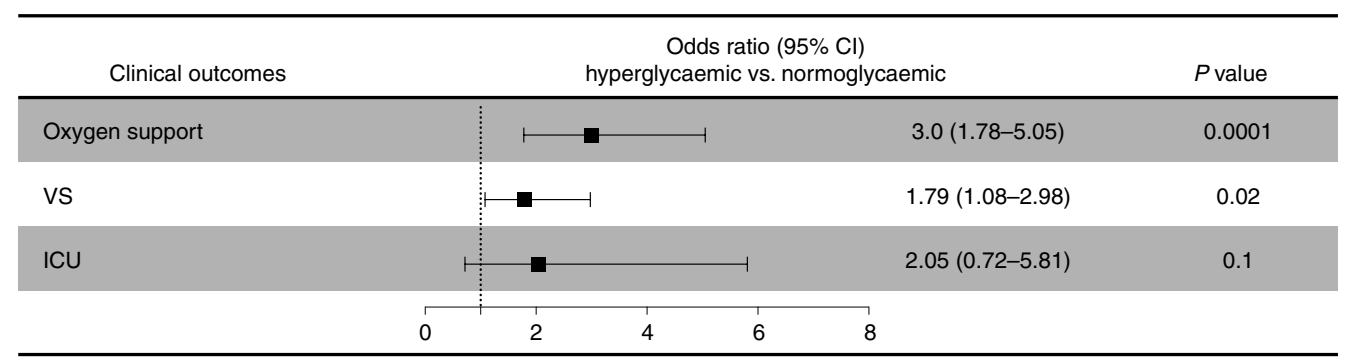


Table 1 | Demographic and clinical characteristics of the study population

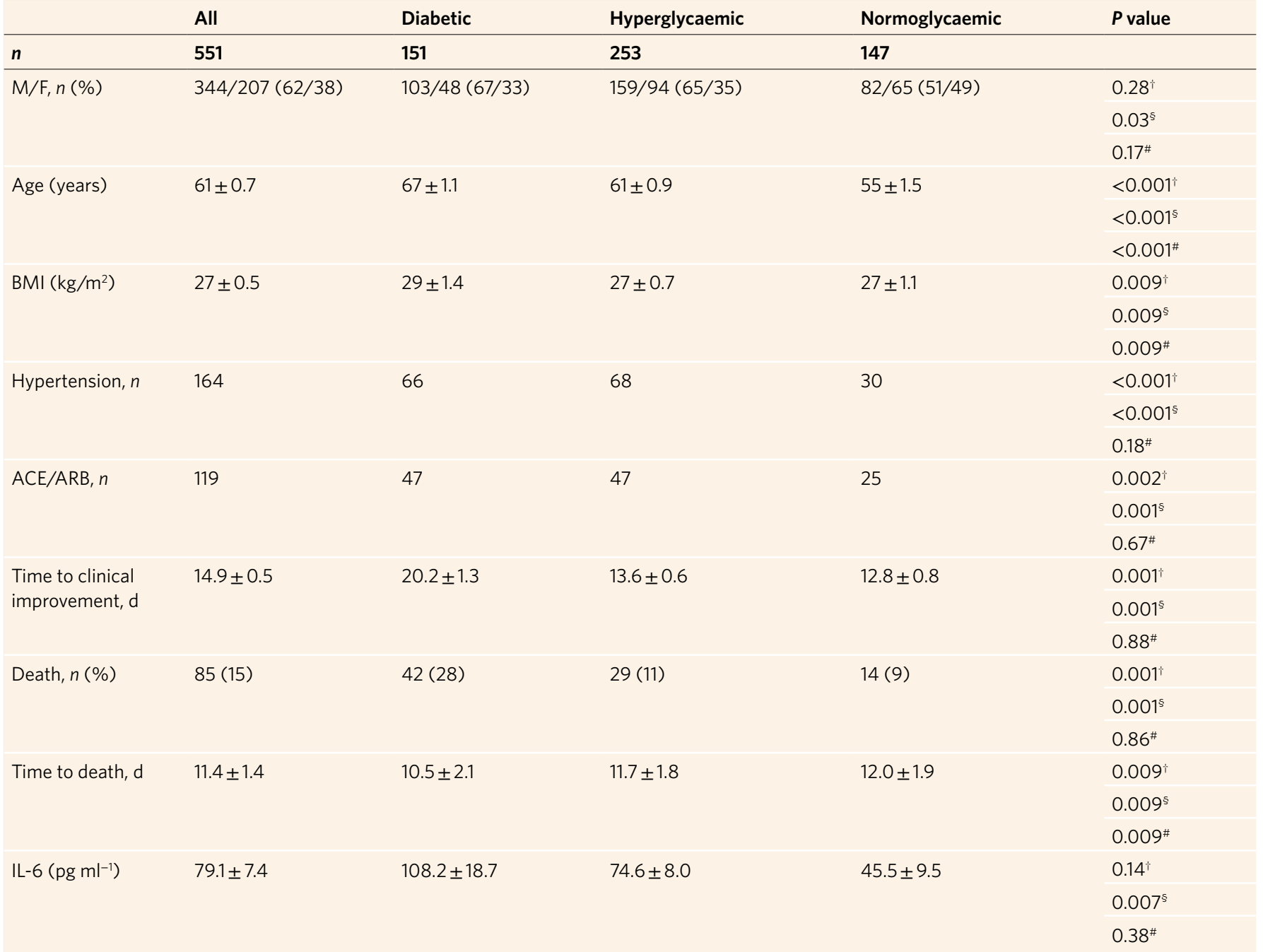

M, males; F, females; $n$, number of patients; ACE, angiotensin-converting enzyme; ARB, angiotensin receptor blocker. Data are expressed as the mean \pm s.e.m. unless otherwise reported. ${ }^{\dagger} P$ Diabetes versus hyperglycaemic; sP Diabetes versus normoglycaemic; \#P Hyperglycaemic versus normoglycaemic.

conditions and after an arginine stimulation test in the subgroup of patients who underwent CGM. Although the arginine stimulation test is not considered to be the standard methodology for assessing beta cell function, several clinical trials have confirmed its reliability and reproducibility when compared to standardized tests ${ }^{26}$. Mean fasting insulin, proinsulin, C-peptide levels, homeostasis model assessment of beta cell dysfunction (HOMA-B) and homeostasis model assessment of insulin resistance (HOMA-IR), but not insulin-to-proinsulin ratio, were significantly higher in patients with COVID-19 as compared to healthy controls (Fig. 3a-f). Interestingly, patients with COVID-19 showed significantly higher maximal acute insulin responses to arginine $\left(\mathrm{AIR}_{\max } ; \mathrm{Fig} .3 \mathrm{~g}\right)$ and higher AUC for insulin and C-peptide in response to the arginine test as compared to healthy controls (Fig. 3h,i). Furthermore, patients who recovered from COVID-19 also showed significantly higher fasting insulin levels, C-peptide levels, HOMA-B and HOMA-IR as compared to healthy controls, with no differences observed in the insulin-to-proinsulin ratio (Fig. 3a-f). Similarly, AIR $_{\max }$ values were significantly higher in patients who recovered from COVID-19 as compared to healthy controls (Fig. 3g). Significantly higher AUC values for insulin, but not for C-peptide, were found in patients who recovered from COVID-19 as compared to healthy controls (Fig. $3 \mathrm{~h}, \mathrm{i})$. Taken together, our data suggest that in patients with COVID-
19 and in patients who have recovered from COVID-19, the hormone profile is altered both under fasting conditions and after an arginine stimulation test, which demonstrates persistent insulin resistance, and that patients with COVID-19 display a similar hormonal profile to individuals with T2D (Fig. 3a-i). Collectively, signs of beta cell hyperstimulation and aberrant functioning were evident in patients with COVID-19, which may eventually exhaust beta cells and lead to their demise ${ }^{27,28}$.

Changes in the secretome are detected after COVID-19. Given the reported higher cytokine levels in SARS-CoV-2-infected patients ${ }^{29-32}$, we evaluated the cytokine profile (that is, secretome) in the serum of patients with COVID-19 or in those who recovered from COVID-19 who had also undergone CGM and an arginine stimulation test. To this end, we performed a multiplexed immunoassay analysis using a Luminex reader, which measures 17 distinct analytes including cytokines and other secreted proteins. Of the 17 analytes assessed, 10 cytokines (interleukin (IL)-1 $\beta$, IL-4, IL-6, IL-7, IL-8, IL-10, IL-13, granulocyte-colony stimulating factor (G-CSF), macrophage inflammatory protein-1 beta (MIP-1 $\beta$ ) and tumour necrosis factor (TNF)) were found to be significantly upregulated in the serum of patients with COVID-19 as compared to healthy controls (Fig. 4a-n and Supplementary Table 2). Notably, 10 of 17 

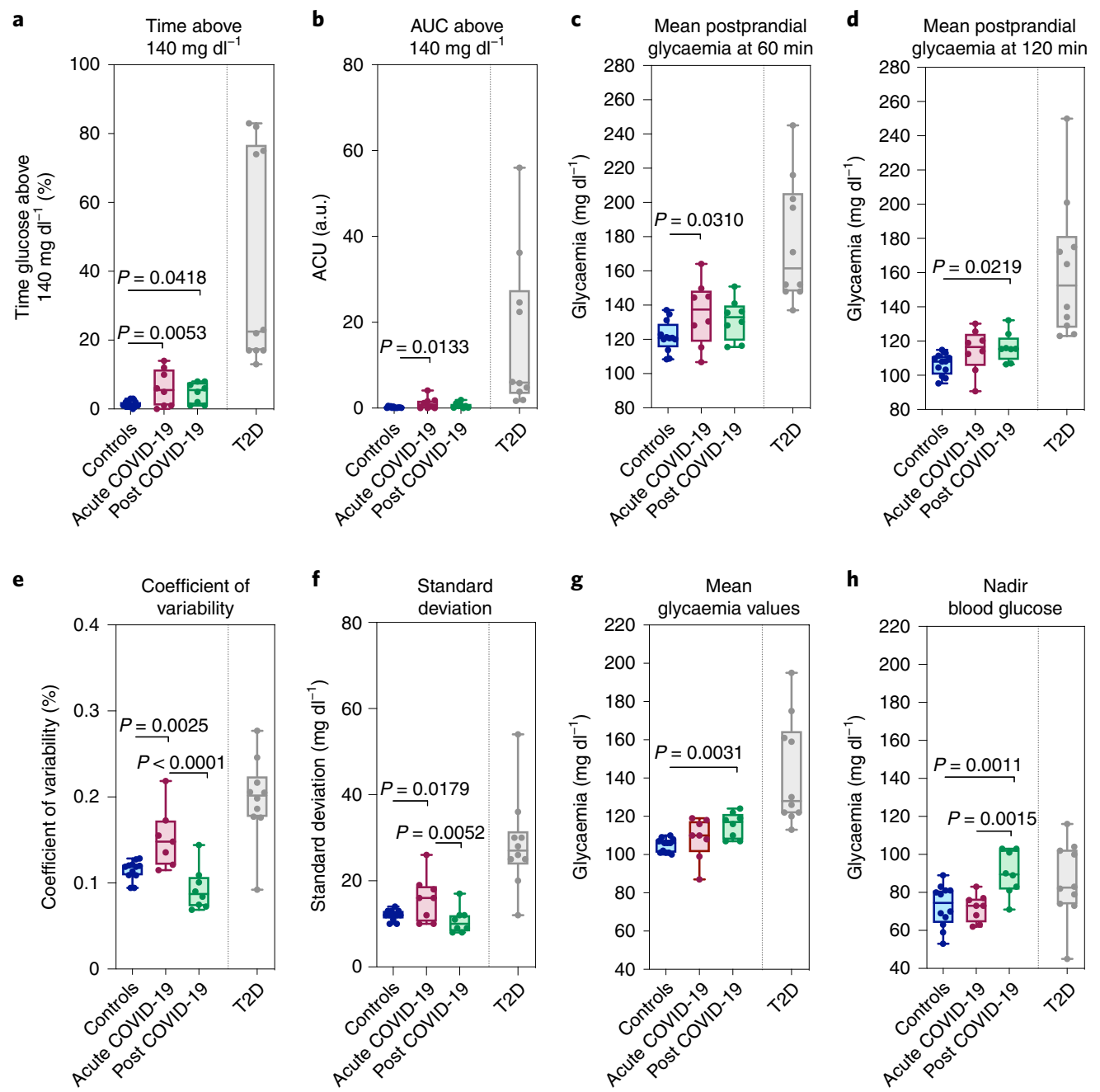

Fig. 2 | Continuous glucose monitoring demonstrated glycaemic abnormalities in patients with COVID-19. a-h, Duration of glycaemia measured above $140 \mathrm{mg} \mathrm{dl}^{-1}(\mathbf{a})$, AUC of glycaemia levels above $140 \mathrm{mg} \mathrm{dl}^{-1}(\mathbf{b})$, mean postprandial glycaemia at $60 \mathrm{~min}$ (c), mean postprandial glycaemia at $120 \mathrm{~min}(\mathbf{d})$, coefficient of variability (e), standard deviation (f), mean glycaemia values ( $(\mathbf{g})$ and nadir blood glucose (h) in healthy controls, in patients with COVID-19 (acute COVID-19), in patients who recovered from COVID-19 (post COVID-19) and in patients with T2D. Data are depicted using box plots and whiskers where the upper and lower bounds of the boxes represent the interquartile ranges. The horizontal line inside each box reflects the median, and the whiskers indicate minimum and maximum values. Each dot represents an individual sample (controls (blue), COVID-19 (maroon) and post COVID-19 (moss)). Ordinary one-way ANOVA test with Bonferroni correction was used when applicable for calculating statistical significance between all groups. Data are representative of $n=12$ samples for controls, $n=8$ (except for $\mathbf{e} ; n=7$ ) for acute COVID-19, $n=8$ for post COVID-19 and $n=10$ for patients with T2D. T2D group (grey) was not included in the statistical analysis and is shown for visual comparison only. a.u., arbitrary units.

analytes examined (IL-1 $\beta$, IL-2, IL-4, IL-7, IL-8, IL-10, IL-13, IL-17, G-CSF and interferon (IFN)- $\gamma$ ) were also increased in the serum of patients who recovered from COVID-19 (Fig. 4a-n). Similar to the pattern observed in patients with COVID-19, several analytes were found to be upregulated in the sera of patients with T2D (Fig. $4 a-n)$. A reduction in IL- 6 and interferon gamma-induced protein 10 (IP-10) levels was observed in the sera of patients who recovered from COVID-19 as compared to healthy controls (for IP-10) and compared to those who had active COVID-19 (for IL-6 and IP-10; Fig. 4d,n). Some of the aforementioned cytokines (that is, IL-2, IL-17 and IFN- $\gamma$ ) appeared exclusively upregulated in patients who recovered from COVID-19 as compared to healthy controls (Fig. 4b,i,l). Moreover, an overall inflammatory score appeared increased in patients with COVID-19 and in those who recovered from COVID-19 (Supplementary Fig. 1a). Inflammatory score correlated with the HOMA-IR, confirming the inflammatory origin of COVID-19-associated insulin resistance (Supplementary Fig. 1b). Altogether, our data demonstrate an altered secretome in patients with COVID-19 and in patients who recovered from COVID-19 (post COVID-19), with an overall increase in many serum cytokine levels but with a different profile from that which is observed in patients with T2D. Overall, CGM, the hormonal profile and the secretome showed many differences in patients with COVID-19, in those who recovered from COVID-19 and in patients with T2D as compared to controls (Fig. 5a-c).

To mechanistically understand whether elevated peripheral levels of cytokines have a clinical impact on glycometabolic control, we evaluated data retrieved from our patient database and observed that patients with pre-existing diabetes had higher peripheral levels of IL-6 at hospital admission as compared to normoglycaemic patients, but not compared to new-onset hyperglycaemic patients (Table 1). In new-onset hyperglycaemic patients with COVID-19, peripheral IL-6 levels correlated with fasting glucose levels (Supplementary Fig. 2a). Interestingly, among patients with new-onset hyperglycaemia, 39 showed particularly elevated peripheral IL-6 levels, and thus received tocilizumab as adjuvant therapy to reduce the COVID-19-associated 
Table 2 | Demographic and clinical characteristics of subgroups analysed for the CGM and arginine tests

n $M / F, n$

\begin{tabular}{l} 
All \\
\hline 35 \\
\hline $21 / 14$
\end{tabular}

Controls Acute COVID-19

1510

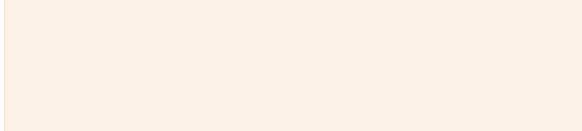

Age, years (mean \pm s.e.m.)

$45.9 \pm 2.1$

$47.2 \pm 3.1$

$43.0 \pm 4.7$

$46.9 \pm 3.8$

T2D

P

$\begin{array}{llll}15 & 10 & 10 & 10\end{array}$

$6 / 4$

0.24

$0.9^{5}$

$0.9^{\#}$

$0.36^{*}$

$0.65^{\xi}$

$0.9^{\psi}$

(n)

4

4

0

0

3

4

0.84

$0.99^{\mathrm{s}}$

$0.90^{\#}$

0.80 *

$0.53^{\xi}$

$0.96^{4}$

Smoking, $n$

13

8

$23.4 \pm 0.6$

$23.3 \pm 0.6$

$24.8 \pm 2.1$

$22.4 \pm 1.5$

$27.2 \pm 0.3$

$0.12^{\dagger}$

$0.12^{5}$

$0.61^{\#}$

$0.9^{*}$

$0.9^{\xi}$

$0.9^{4}$

$0.21^{\dagger}$

$0.41^{\mathrm{s}}$

$0.68^{\#}$

$0.9^{*}$

$0.62^{\xi}$

$0.9^{\psi}$

BMI (mean \pm s.e.m.)

$23.4 \pm 0.6$

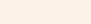

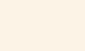

$0.72^{\dagger}$

$0.94^{5}$

$0.014^{\#}$

$0.53^{*}$

$0.40^{\xi}$

$0.020^{\psi}$

Hypertension (\%)

1

0

0

$0.9^{\dagger}$

$0.9^{\text {s }}$

$0.022^{\#}$

$0.9^{*}$

$0.032^{\xi}$

$0.032 \%$

$<0.001$

$<0.001$

Baseline clinical score (mean \pm s.e.m.)

$34.0 \pm 5.7-$

$22.9 \pm 4.1$

$62.0 \pm 3.2$

$2.0 \pm 0.0$

$0.55^{*}$

0.06

$0.9^{*}$

$0.33^{*}$

$0.001^{*}$

$0.9^{*}$

Antibiotics

6

6

Diabetes-associated therapies, $n$

Metformin

Others

0

0

0

0

0

No therapies

LMWH, low-molecular-weight heparin. ${ }^{\dagger}$ P Controls versus acute COVID-19; ${ }^{5}$ P Controls versus post COVID-19; \#P Controls versus T2D; $A$ cute COVID-19 versus post COVID-19; ${ }^{\xi}$ Acute COVID-19 versus T2D; \% Post COVID-19 versus T2D 

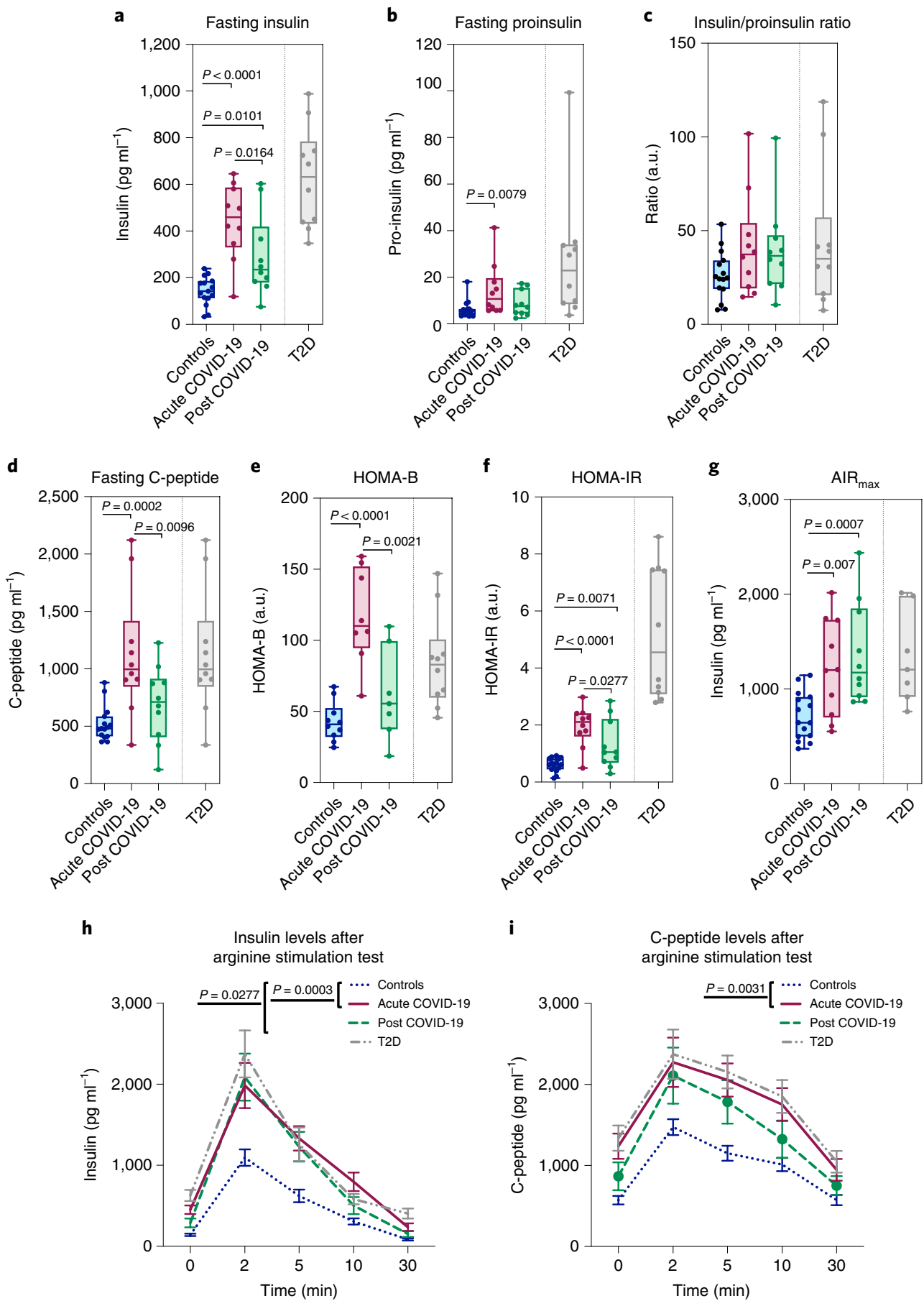

Fig. 3 | Persistent insulin resistance and beta cell dysfunction are evident in patients with COVID-19. a-f, Mean fasting insulin (a), mean fasting proinsulin (b), fasting insulin-to-proinsulin ratio (c), fasting C-peptide levels (d), HOMA-B (e) and HOMA-IR (f) are shown for healthy controls, for patients with COVID-19 (acute COVID-19), for patients who recovered from COVID-19 (post COVID-19) and for patients with T2D. $\mathbf{g}-\mathbf{i}$, AIR max $_{\text {( }}$ (g) and mean AUC of insulin and C-peptide after arginine test ( $\mathbf{h}$ and $\mathbf{i})$ are shown for healthy controls, for the acute COVID-19 group or for the post-COVID-19 group, and for individuals with T2D. Data are depicted using box plots and whiskers where the upper and lower bounds of the boxes represent the interquartile ranges. The horizontal line inside each box reflects the median, and the whiskers indicate minimum and maximum values. Each dot represents an individual sample (controls (blue), COVID-19 (maroon) and post COVID-19 (moss). Ordinary one-way ANOVA test with Bonferroni correction was used when applicable for calculating statistical significance between all groups. Data are representative of $n=15$ (except for $\mathbf{e} ; n=10)$ samples analysed for controls, $n=10$ (except for $\mathbf{e} ; n=8$ ) for acute COVID-19, $n=10$ (except for $\mathbf{e} ; n=7$ ) for post COVID-19 and $n=10$ (except for $\mathbf{g} ; n=7$ ) for individuals with T2D. T2D group (grey) was not included in the statistical analysis and is included for visual comparison only.

inflammation. Patients with tocilizumab-treated COVID-19 who had new-onset hyperglycaemia showed a greater reduction in glycaemic levels at the time of hospital discharge compared to patients who did not receive tocilizumab (Supplementary Fig. 2b). This exploratory study requires further investigation to confirm a link between cytokine levels and glycometabolic abnormalities. 
a

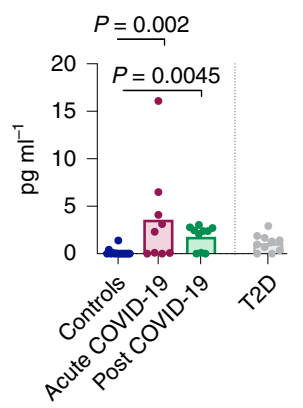

f

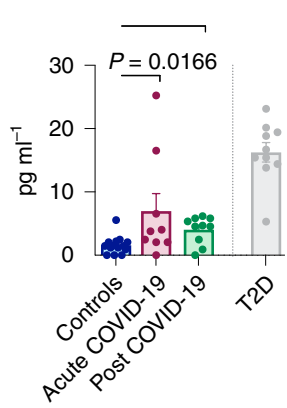

k

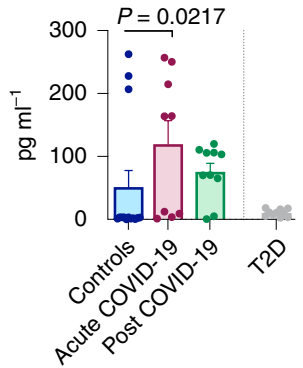

b

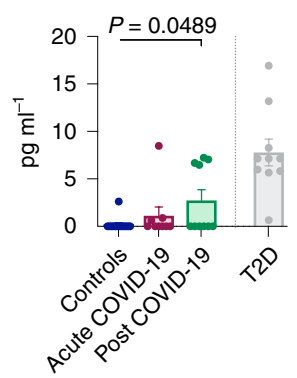

g

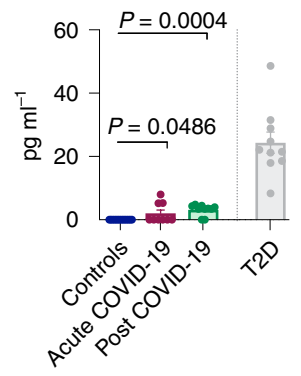

I

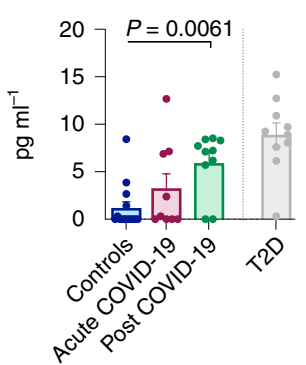

c

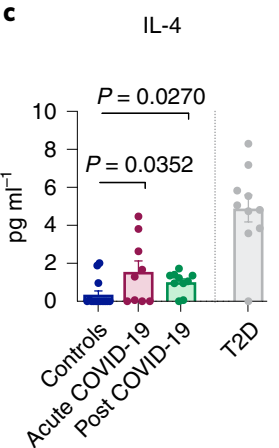

h

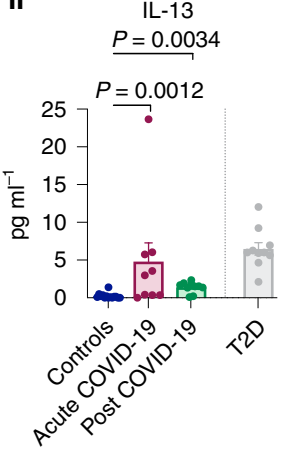

m

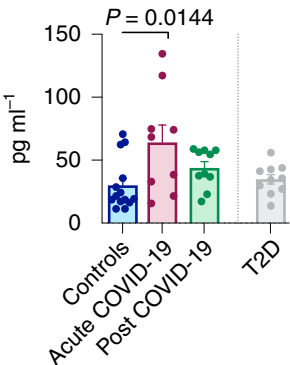

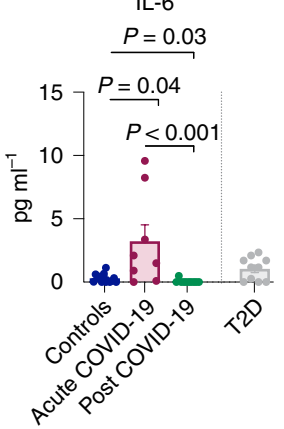

i

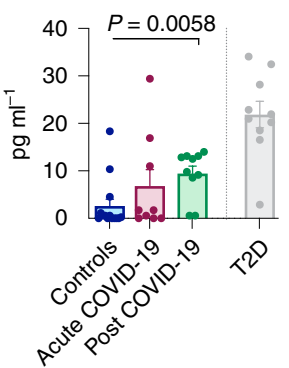

n

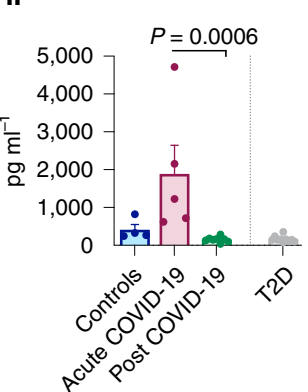

e

IL-7
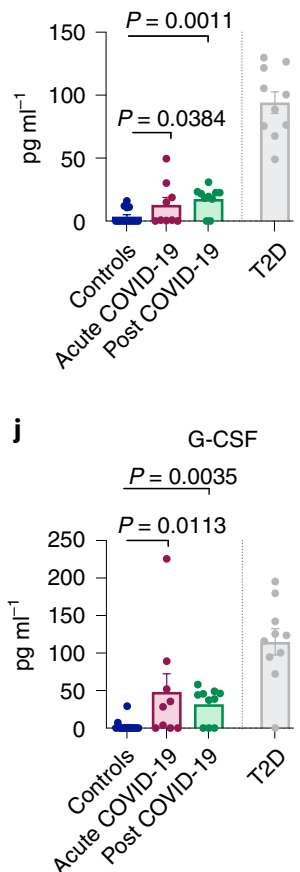

Fig. 4 | Changes in the secretome are detected long after recovery from COVID-19. a-n, The peripheral levels of 14 circulating cytokines (IL-1 $\beta$, IL-2, IL-4, IL-6, IL-7, IL-8, IL-10, IL-13, IL-17, G-CSF, MIP-1 $\beta$, IFN- $\gamma$, TNF and IP-10) were assessed by a Luminex assay using the sera of healthy controls, patients with COVID-19 (acute COVID-19) and patients who recovered from COVID-19 (post COVID-19) and patients with T2D. Data are represented as scatterplots showing the mean \pm s.e.m. Each dot represents an individual sample (controls (blue), COVID-19 (maroon) and post COVID-19 (moss)). Statistical significance was determined by unpaired Kruskal-Wallis test. Data are representative of $n=14$ (except for $\mathbf{n} ; n=4$ ) samples analysed for controls, $n=9$ (except for $\mathbf{n} ; n=5$ ) for patients with acute COVID-19, $n=10$ (except for $\mathbf{n} ; n=8$ ) for patients post COVID-19 and $n=10$ for patients with T2D. T2D group (grey) was not included in the statistical analysis and is shown for visual comparison only.

\section{Discussion}

It has recently become evident that a mutual interplay between COVID-19 and diabetes exists, involving a complex pathophysiological feature underlying hyperglycaemia and overall glycometabolic distress ${ }^{16,33-36}$. Indeed, clinical evidence has suggested that COVID-19 may severely reduce life expectancy in patients with $\mathrm{T} 2 \mathrm{D}^{34,37-39}$. In our study, we demonstrated the presence of new-onset hyperglycaemia, insulin resistance and beta cell hyperstimulation in patients with COVID-19 without a history of diabetes. This effect appears to be mediated by the abnormal secretome, which remained altered long after remission of the disease. While metabolic alterations have been described as consequences of other viral infections ${ }^{40,41}$, COVID-19 may induce an inflammatory state resembling that which is observed in $\mathrm{T}_{2} \mathrm{D}^{33,35,36}$ but which is more exacerbated; in the long term, these effects may lead to beta cell exhaustion and worsening of diabetes caused by islet hyperstimulation and glucose toxicity ${ }^{42-50}$. Indeed, newly hyperglycaemic patients treated with tocilizumab showed a significant reduction in glycaemic levels at the time of their discharge from the hospital as compared to patients who did not receive tocilizumab. The negative results of the latest placebo-controlled trial with tocilizumab, which enrolled moderately ill hospitalized patients, suggest that our results are possibly metabolic/endocrine related rather than disease related ${ }^{51}$. The percentage of patients with hyperglycaemia is surprisingly high among patients admitted to the hospital for COVID-19-related pneumonia. Patients without a history or diagnosis of diabetes and admitted to the hospital with normal glycated haemoglobin showed varying degrees of glycometabolic impairment and beta cell dysfunction. This is notable in view of the elevated mortality rates that we and others have observed in patients with COVID19 who presented with hyperglycaemia ${ }^{9,38,52,53}$. 


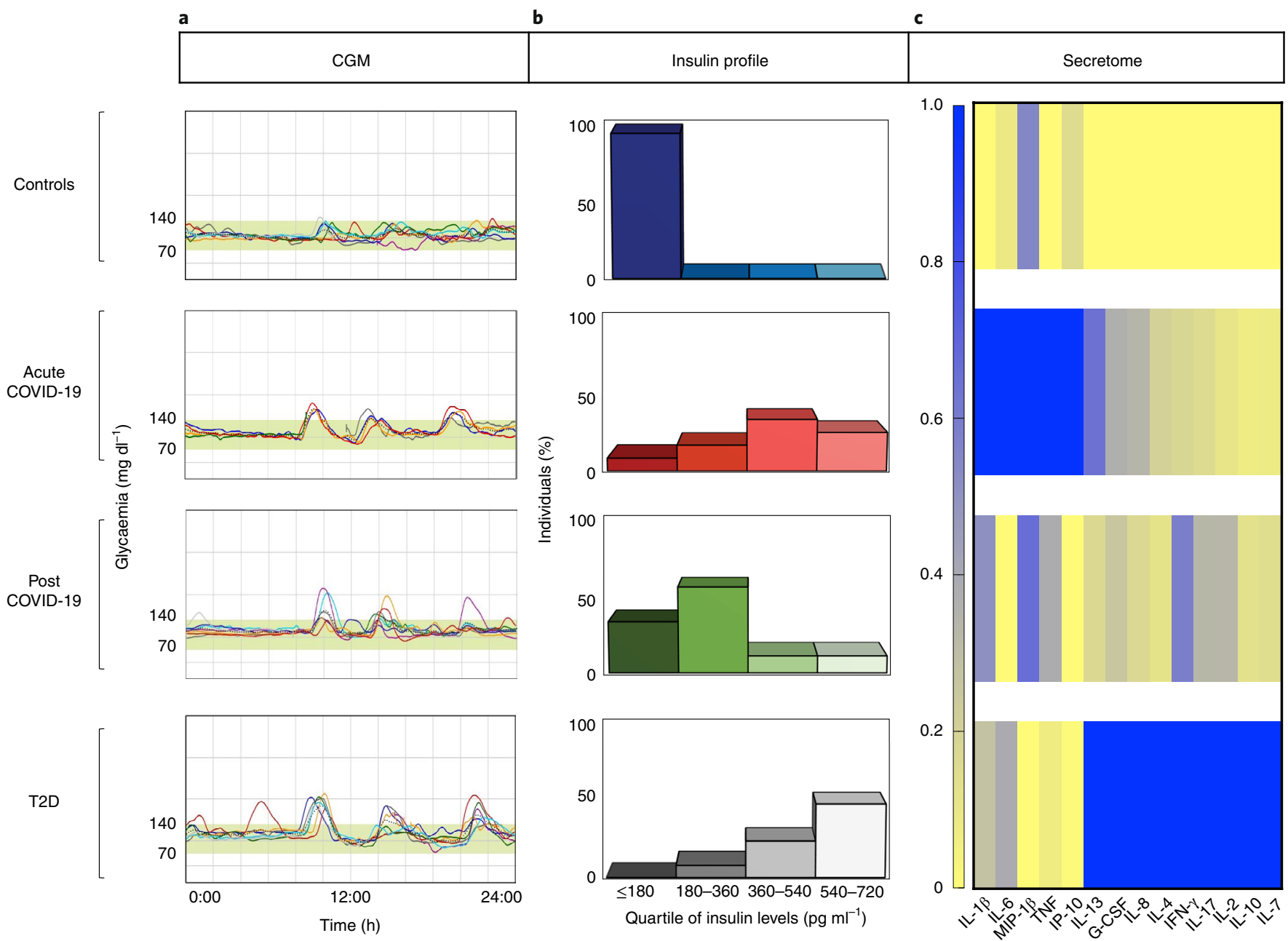

Fig. 5 | Evidence of glycometabolic, hormonal and secretome abnormalities in patients with COVID-19. a-c, Comparative schematic/analysis showing abnormalities in CGM (a), insulin levels (b) and secretome profile (c) in patients with COVID-19 (acute COVID-19), in those who recovered from COVID19 (post COVID-19) and in patients with T2D, demonstrating similarities with those found in patients with T2D. Data in c are represented as colour-coded values showing the average of serum cytokine mean-normalized levels in each group, normalized across groups.

In this study, we observed glycaemic alterations not only in the acute phase of COVID-19 but also long after remission of the disease. We acknowledge that our study has limitations: firstly, we recognize the potentially inadequate sample size, which may limit the conclusions that can be drawn from these data. A sample of 551 patients may allow for reasonable power for the study, but the subgroup analysis may be underpowered because of the small sample size. We further acknowledge that the participants included in this subgroup analysis displayed lower age and body mass index (BMI) as compared to patients included in the entire study. Participant recruitment into study groups was performed consecutively as patients were admitted to the hospital, and recruitment was thus devoid of any bias; indeed, no statistical differences were evident when comparing demographic parameters within the subgroups. Furthermore, due to increased morbidity and mortality associated with COVID-19 in older patients, the average age of patients eligible for and included in this study was lower than that of the general population. Lastly, patients were recruited from a single, large academic hospital (ASST FBF-Sacco Milan, Presidio Sacco), such that the study results may be subject to hospital selection bias.

Interestingly, one of the major findings of our work is that CGM allowed for detection of alterations in glucose homeostasis not otherwise detectable by self-measurement of fasting blood glucose $\mathrm{e}^{54}$.
In accordance with this observation, we also reported alterations in the hormone profile, both at basal levels and after stimulation testing, with higher insulin, proinsulin and C-peptide levels in patients with COVID-19 (acute COVID-19) and in patients who recovered from COVID-19 (post COVID-19) as compared to healthy controls. Our observations further indicate that COVID-19 disrupts insulin signalling and beta cell function, in addition to the previously reported long-term effects on cardiovascular, neurological and renal function ${ }^{55,56}$.

Our data suggest that a proinflammatory milieu initiated by a cytokine storm, in which IL-6 plays a primary albeit not exclusive role, induces insulin resistance and beta cell dysfunction as shown for $\mathrm{T} 2 \mathrm{D}^{57,58}$. Any bias associated with pharmacologic therapy can likely be excluded, because few patients received steroids and hydroxychloroquine during the course of the disease. To conclude, in the present study, we showed that the cytokine profile in the sera of patients with COVID-19 and survivors is markedly different from that of controls and that the observed hyperglycaemia, insulin resistance and beta cell dysfunction might be due to the proinflammatory milieu initiated by a cytokine storm. This study demonstrates that SARS-CoV-2 induces insulin resistance and disrupts proper beta cell function, which can result in clinically evident hyperglycaemia detectable even in the post-acute phase. Our findings sug- 
gest the persistence of aberrant glycometabolic control long after recovery from the disease. This persistence should be investigated in larger cohort and its effect on clinical symptoms and sequalae should be carefully addressed.

\section{Methods}

Study design and outcomes. All research studies and analysis reported in this paper were performed in accordance with the local Ethical Research Committee of Milan (Comitato Etico Milano Area 1), which granted the study approval (no. 2020/ST/167). The written informed consent and ethical committee approval covered all experimental analysis performed and reported in this study.

Data from patients admitted for SARS-CoV-2 acute infection at ASST FBF-Sacco Milan, Presidio Sacco, from 1 February 2020 to 15 May 2020 were collected. Confirmed COVID-19 was defined as detection of SARS-CoV-2 by RT-PCR in respiratory samples. Patient baseline clinical score was defined on the basis of a modified ordinal score comprising seven major points as follows and as previously reported ${ }^{34}$ : (1) not hospitalized with resumption of normal activities; (2) not hospitalized, but unable to resume normal activities; (3) hospitalized, not requiring supplemental oxygen; (4) hospitalized, requiring supplemental oxygen; (5) hospitalized, requiring non-invasive mechanical ventilation; (6) hospitalized, requiring invasive mechanical ventilation or extracorporeal membrane oxygenation; and (7) death. All clinical data were extracted from patient electronic medical reports with regard to baseline demographic distributions, clinical data, laboratory data, management and outcome data (Table 1). Glycaemia levels were evaluated for each patient at three different time points: admission to the emergency room, in-hospital stay and discharge from hospital. HbAlc levels during in-hospital stays were also recorded where available. Patients were classified as having previously known diabetes based on a known history of diabetes or based on an antidiabetic drug regimen. Patients were classified as having newly diagnosed diabetes based on the ADA criteria ${ }^{25}$. Patients were classified as hyperglycaemic based on a blood glucose measurement recorded between 100 and $199 \mathrm{mg} \mathrm{dl}^{-1}$ or two blood glucose measurements of $>100 \mathrm{mg} \mathrm{dl}^{-1}$ and $<126 \mathrm{mg} \mathrm{dl}^{-1}$. Patients were classified as normoglycaemic in the absence of a previously known history of diabetes or hyperglycaemia, as well as if they displayed normal levels of glycaemia and HbAlc according to the ADA criteria ${ }^{2}$. Groups of consecutive patients with COVID-19 or patients who recovered from COVID-19 infection, based on the clinical evaluation by the Infectious Disease and Respiratory Division of ASST FBF-Sacco Milan and based on a prior positive COVID-19 test, and who were normoglycaemic with no history of diabetes or IFG (impaired fasting glucose) or IGT (impaired glucose tolerance), were enrolled within the original group of 551 patients and compared with a paired group of healthy controls. We also included a small group of patients with T2D as a further control. Patients with T2D were treated with metformin and/or were following dietary restrictions. Patients underwent glucose monitoring using a retrospective professional CGM device ('Continuous glucose monitoring') and an intravenous arginine acute stimulation insulin secretion test ${ }^{26}$ ('Hormone level assessment'). Inclusion criteria were defined by recruitment of male and female participants, aged $>18$ years and $<80$ years with normoglycaemia, no history of diabetes or IFG/IGT and no use of drugs with known effects on glucose metabolism. Exclusion criteria were: age $<18$, a history of diabetes or IFG or impaired glucose tolerance IGT, use of drugs with known effects on glucose metabolism and pregnancy.

Continuous glucose monitoring. A group of participants among normoglycaemic individuals including healthy controls $(n=12)$, patients with COVID-19 $(n=8)$ and patients who recovered from COVID-19 $(n=8)$ enrolled within the original group of 551 patients, underwent complete glycaemic profiling via 7 -d professional retrospective CGM (Medtronic Envision Pro CGM, Medtronic Minimed). The system consists of a fully calibrated device composed of an EC-approved Envision Sensor, Envision Recorder, Envision Pro Application and CareLink Pro Software. Both patients and clinical site staff were blinded to CGM results during the study. Patient informed consent on CareLink Software for Professional CGM Systems was obtained for each registration. Mean blood glucose, estimated HbAlc, peak and nadir blood glucose, time above $140 \mathrm{mg} \mathrm{dl}^{-1}$, AUC above $140 \mathrm{mg} \mathrm{dl}^{-1}$ limit, mean postprandial glycaemia values at 60 and $120 \mathrm{~min}$, standard deviation and coefficient of variability values were evaluated during the registration period.

Hormone level assessment. Insulin and C-peptide secretion was determined using an intravenous arginine stimulation test as previously described ${ }^{14}$. An intravenous catheter was inserted into the antecubital vein of the patient's arm. The sampling catheter was kept patent by slow infusion of $0.9 \%$ saline when not used. Baseline samples were taken at $0 \mathrm{~min}$. A maximal stimulating dose of arginine hydrochloride $(5 \mathrm{~g})$ was then injected intravenously for $45 \mathrm{~s}$. Samples were taken at $+2,+5,+10$ and +30 min. For fasting glucose, $\operatorname{AIR}_{\max }$ was determined as the mean of the three highest insulin values from minutes 2,5 and 10 subtracted from the baseline insulin. The fasting insulin-to-proinsulin ratio was calculated as an index of beta cell function as previously described ${ }^{59}$. Insulin resistance was calculated using the HOMA-IR formula: fasting insulin $\left(\mathrm{mIU} \mathrm{ml}^{-1}\right)$ fasting glucose $\left.\left(\mathrm{mmol} \mathrm{l}^{-1}\right) / 22.5\right)^{60}$. HOMA-B index was calculated using the following formula: $20 \times$ fasting insulin $\left(\mu \mathrm{IU} \mathrm{ml}{ }^{-1}\right) /$ fasting glucose $\left(\mathrm{mmol} \mathrm{ml}^{-1}\right)-3.5$. (ref. $\left.{ }^{61}\right)$.
Inflammatory score. Each plasma cytokine value was stratified into quintiles to determine cut-off points and assign a score ranging from 0 , which was assigned to the lowest quintile, to 4 , which was assigned to the highest quintiles ${ }^{58}$.

Biochemical analyses. Fasting serum samples of patients and controls were collected at the designated time points and frozen at $-80^{\circ} \mathrm{C}$ for biochemical evaluation. Baseline levels of the analytes G-CSF, IFN- $\gamma$, IL-1 $\beta$, IL-2, IL-4, IL-5, IL-6, IL-7, IL-8, IL-10, IL-12, IL-13, IL-17A, MCP-1, MIP-1 $\beta$, TNF and IP-10 were assessed in the sera of patients and controls by a magnetic microsphere-based Bio-Plex Pro Human Cytokine 17-plex immunoassay (M5000031YV) on a Bio-Plex 200 system (both from Bio-Rad) according to the manufacturer's instructions. Serum proinsulin (Mercodia, 10-1118-01) and HbA1c (Aviva System Biology, OKEH00660) levels at baseline were assessed by ELISA using commercial kits according to the manufacturers' recommendations. Basal glycaemia was assessed using a colorimetric assay (Life Technologies, EIAGLUC) on serum samples collected in tubes containing potassium oxalate/sodium fluoride. Finally, serum samples obtained from participants who underwent an arginine stimulation test were collected at each arginine test time point (T0-T4) and were evaluated for insulin, C-peptide and glucagon concentrations using the Bio-Plex Pro Human Diabetes 10-Plex Assay kit (171A7001M) and a Bio-Plex 200 reader (Bio-Rad) according to the manufacturer's instructions.

Statistical analysis. Continuous variables are presented as means with standard errors, and categorical variables are presented as proportions. We used independent sample $t$-tests to compare continuous variables and a chi-squared test/Fisher's exact test to compare categorical variables. For multiple comparisons, one-way or two-way ANOVA followed by Bonferroni post hoc test between the group of interest and all other groups was used, when applicable. Spearman correlation analysis was performed to assess relations among mortality and other population characteristics. A log-rank test was used to compare survival curves. Two-tailed $P$ values of less than 0.05 were considered statistically significant. Multivariable logistic regression was used to model the relationships between risk factors and clinical outcomes (Stata version 12; StataCorp). Age and sex were included in the multivariable logistic regression analysis performed for each clinical outcome. A log-rank (Mantel-Cox) test was used in the time to clinical endpoint analysis among groups (GraphPad Prism version 8.4.3; GraphPad Software). Spearman's correlation was used to examine the association between glycaemia and peripheral IL-6 levels. Two-tailed $P$ values of less than 0.05 were considered statistically significant. Microsoft Excel version 16.30 was used to generate graphs related to Fig. $5 \mathrm{~b}$.

Power analysis. Sample size was set at 15 in the control group and 10 in the other subgroups, to provide the study with $80 \%$ power to detect a difference of at least $15 \%$ in the mean AUC insulin response to $5 \mathrm{~g}$ intravenous arginine between the groups, with a significance level of $\alpha=0.05$, given that the mean AUC insulin response observed in participants with normal glucose tolerance undergoing an arginine test is $1,083 \pm 132 \mathrm{pmol} \mathrm{l}^{-1}$ (ref. ${ }^{62}$ ).

Reporting Summary. Further information on research design is available in the Nature Research Reporting Summary linked to this article.

\section{Data availability}

Source data are provided with this paper. All other data that support the findings of this study are available from the corresponding author upon request.

Received: 2 December 2020; Accepted: 12 May 2021;

Published online: 25 May 2021

\section{References}

1. Xu, B., Kraemer, M. U. G. \& Open COVID-19 Data Curation Group. Open access epidemiological data from the COVID-19 outbreak. Lancet Infect. Dis. 20, 534 (2020)

2. Tay, M. Z., Poh, C. M., Renia, L., MacAry, P. A. \& Ng, L. F. P. The trinity of COVID-19: immunity, inflammation and intervention. Nat. Rev. Immunol. 20, 363-374 (2020).

3. Hu, B., Guo, H., Zhou, P. \& Shi, Z. L. Characteristics of SARS-CoV-2 and COVID-19. Nat. Rev. Microbiol. https://doi.org/10.1038/s41579-020-00459-7 (2020).

4. Deng, S. Q. \& Peng, H. J. Characteristics of and public health responses to the coronavirus disease 2019 outbreak in China. J. Clin. Med. https://doi. org/10.3390/jcm9020575 (2020).

5. Chen, N. et al. Epidemiological and clinical characteristics of 99 cases of 2019 novel coronavirus pneumonia in Wuhan, China: a descriptive study. Lancet 395, 507-513 (2020)

6. Fadini, G. P., Morieri, M. L., Longato, E. \& Avogaro, A. Prevalence and impact of diabetes among people infected with SARS-CoV-2. J. Endocrinol. Invest. 43, 867-869 (2020). 
7. Yang, J. K. et al. Plasma glucose levels and diabetes are independent predictors for mortality and morbidity in patients with SARS. Diabet. Med. 23, 623-628 (2006).

8. Yang, J. K., Lin, S. S., Ji, X. J. \& Guo, L. M. Binding of SARS coronavirus to its receptor damages islets and causes acute diabetes. Acta Diabetol. 47, 193-199 (2010).

9. Apicella, M. et al. COVID-19 in people with diabetes: understanding the reasons for worse outcomes. Lancet Diabetes Endocrinol. 8, 782-792 (2020).

10. Steenblock, C., et al. Beta cells from patients with COVID-19 and from isolated human islets exhibit ACE2, DPP4 and TMPRSS2 expression, viral infiltration and necroptotic cell death. Preprint at Res. Sq. https://doi. org/10.21203/rs.3.rs-88524/v1 (2020).

11. Kusmartseva, I. et al. Expression of SARS-CoV-2 entry factors in the pancreas of normal organ donors and individuals with COVID-19. Cell Metab. 32, 1041-1051 (2020).

12. Yang, L. et al. A human pluripotent stem cell-based platform to study SARS-CoV-2 tropism and model virus infection in human cells and organoids. Cell Stem Cell 27, 125-136 (2020).

13. Liu, F. et al. ACE2 expression in pancreas may cause pancreatic damage after SARS-CoV-2 infection. Clin. Gastroenterol. Hepatol. 18, 2128-2130 (2020).

14. Ding, Y. et al. Organ distribution of severe acute respiratory syndrome (SARS) associated coronavirus (SARS-CoV) in SARS patients: implications for pathogenesis and virus transmission pathways. J. Pathol. 203, 622-630 (2004)

15. Muller, J. A. et al. SARS-CoV-2 infects and replicates in cells of the human endocrine and exocrine pancreas. Nat. Metab. 3, 149-165 (2021).

16. Bornstein, S. R. et al. Practical recommendations for the management of diabetes in patients with COVID-19. Lancet Diabetes Endocrinol. 8, 546-550 (2020)

17. Dungan, K. M., Braithwaite, S. S. \& Preiser, J. C. Stress hyperglycaemia. Lancet 373, 1798-1807 (2009).

18. Dave, G. S. \& Kalia, K. Hyperglycemia induced oxidative stress in type-1 and type-2 diabetic patients with and without nephropathy. Cell Mol. Biol. 53, 68-78 (2007)

19. de Carvalho Vidigal, F., Guedes Cocate, P., Goncalves Pereira, L. \& de Cassia Goncalves Alfenas, R. The role of hyperglycemia in the induction of oxidative stress and inflammatory process. Nutr. Hosp. 27, 1391-1398 (2012).

20. Fabbri, A. et al. Stress hyperglycemia and mortality in subjects with diabetes and sepsis. Crit. Care Explor. 2, e0152 (2020).

21. Niewczas, M. A. et al. A signature of circulating inflammatory proteins and development of end-stage renal disease in diabetes. Nat. Med. 25, 805-813 (2019).

22. Folli, F. et al. Proteomics reveals novel oxidative and glycolytic mechanisms in type 1 diabetic patients' skin which are normalized by kidney-pancreas transplantation. PLoS ONE 5, e9923 (2010).

23. Bassi, R. \& Fiorina, P. Impact of islet transplantation on diabetes complications and quality of life. Curr. Diab. Rep. 11, 355-363 (2011).

24. La Rocca, E. et al. Patient survival and cardiovascular events after kidneypancreas transplantation: comparison with kidney transplantation alone in uremic IDDM patients. Cell Transplant. 9, 929-932 (2000).

25. American Diabetes Association. Introduction: standards of medical care in diabetes-2020. Diabetes Care 43 (Suppl. 1), S1-S2 (2020).

26. Shankar, S. S. et al. Standardized mixed-meal tolerance and arginine stimulation tests provide reproducible and complementary measures of beta cell function: results from the foundation for the National Institutes of Nealth biomarkers consortium investigative series. Diabetes Care 39, 1602-1613 (2016).

27. Erion, K. \& Corkey, B. E. Beta cell failure or beta cell abuse? Front. Endocrinol. 9, 532 (2018)

28. Weir, G. C. \& Bonner-Weir, S. Five stages of evolving beta cell dysfunction during progression to diabetes. Diabetes 53, S16-S21 (2004).

29. Wang, J., Jiang, M., Chen, X. \& Montaner, L. J. Cytokine storm and leukocyte changes in mild versus severe SARS-CoV-2 infection: review of 3,939 COVID-19 patients in China and emerging pathogenesis and therapy concepts. J. Leukoc. Biol. 108, 17-41 (2020).

30. Ruan, Q., Yang, K., Wang, W., Jiang, L. \& Song, J. Clinical predictors of mortality due to COVID-19 based on an analysis of data of 150 patients from Wuhan, China. Intensive Care Med. 46, 846-848 (2020).

31. Vaninov, N. In the eye of the COVID-19 cytokine storm. Nat. Rev. Immunol. 20, 277 (2020).

32. Mehta, P. et al. COVID-19: consider cytokine storm syndromes and immunosuppression. Lancet 395, 1033-1034 (2020).

33. Solerte, S. B., Di Sabatino, A., Galli, M. \& Fiorina, P. Dipeptidyl peptidase-4 (DPP4) inhibition in COVID-19. Acta Diabetol. 57, 779-783 (2020).

34. Solerte, S. B. et al. Sitagliptin treatment at the time of hospitalization was associated with reduced mortality in patients with type 2 diabetes and COVID-19: a multicenter, case-control, retrospective, observational study. Diabetes Care https://doi.org/10.2337/dc20-1521 (2020).

35. Hollstein, T. et al. Autoantibody-negative insulin-dependent diabetes mellitus after SARS-CoV-2 infection: a case report. Nat. Metab. 2, 1021-1024 (2020).
36. Naguib, M. N. et al. New-onset diabetes with diabetic ketoacidosis in a child with multisystem inflammatory syndrome due to COVID-19. J. Pediatr. Endocrinol. Metab. https://doi.org/10.1515/jpem-2020-0426 (2020).

37. Obukhov, A. G. et al. SARS-CoV-2 infections and ACE2: clinical outcomes linked with increased morbidity and mortality in individuals with diabetes. Diabetes 69, 1875-1886 (2020).

38. Sardu, C. et al. Outcomes in patients with hyperglycemia affected by COVID-19: can we do more on glycemic control? Diabetes Care 43, 1408-1415 (2020).

39. Cole, S. A., Laviada-Molina, H. A., Serres-Perales, J. M., Rodriguez-Ayala, E. \& Bastarrachea, R. A. The COVID-19 pandemic during the time of the diabetes pandemic: likely fraternal twins? Pathogens https://doi.org/10.3390/ pathogens 9050389 (2020).

40. Krogvold, L. et al. Detection of a low-grade enteroviral infection in the islets of langerhans of living patients newly diagnosed with type 1 diabetes. Diabetes 64, 1682-1687 (2015).

41. Laitinen, O. H. et al. Coxsackievirus B1 is associated with induction of betacell autoimmunity that portends type 1 diabetes. Diabetes 63, 446-455 (2014).

42. Hotamisligil, G. S. Inflammation, metaflammation and immunometabolic disorders. Nature 542, 177-185 (2017).

43. Monroy, A. et al. Impaired regulation of the TNF- $\alpha$-converting enzyme/tissue inhibitor of metalloproteinase 3 proteolytic system in skeletal muscle of obese type 2 diabetic patients: a new mechanism of insulin resistance in humans. Diabetologia 52, 2169-2181 (2009).

44. Joya-Galeana, J. et al. Effects of insulin and oral antidiabetic agents on glucose metabolism, vascular dysfunction and skeletal muscle inflammation in type 2 diabetic subjects. Diabetes Metab. Res. Rev. 27, 373-382 (2011).

45. DeFronzo, R. A. Pathogenesis of type 2 (non-insulin dependent) diabetes mellitus: a balanced overview. Diabetologia 35, 389-397 (1992).

46. Mauvais-Jarvis, F. et al. A model to explore the interaction between muscle insulin resistance and beta cell dysfunction in the development of type 2 diabetes. Diabetes 49, 2126-2134 (2000).

47. Marzban, L. New insights into the mechanisms of islet inflammation in type 2 diabetes. Diabetes 64, 1094-1096 (2015).

48. Donath, M. Y., Boni-Schnetzler, M., Ellingsgaard, H., Halban, P. A. \& Ehses, J. A. Cytokine production by islets in health and diabetes: cellular origin, regulation and function. Trends Endocrinol. Metab. 21, 261-267 (2010).

49. Butcher, M. J. et al. Association of proinflammatory cytokines and islet resident leucocytes with islet dysfunction in type 2 diabetes. Diabetologia 57, 491-501 (2014)

50. Eguchi, K. \& Nagai, R. Islet inflammation in type 2 diabetes and physiology. J. Clin. Invest. 127, 14-23 (2017)

51. Stone, J. H. et al. Efficacy of tocilizumab in patients hospitalized with COVID-19. N. Engl. J. Med. https://doi.org/10.1056/NEJMoa2028836 (2020).

52. D’Addio, F. et al. Islet transplantation stabilizes hemostatic abnormalities and cerebral metabolism in individuals with type 1 diabetes. Diabetes Care 37, 267-276 (2014).

53. Astorri, E., Fiorina, P., Gavaruzzi, G., Astorri, A. \& Magnati, G. Left ventricular function in insulin-dependent and in non-insulin-dependent diabetic patients: radionuclide assessment. Cardiology 88, 152-155 (1997)

54. Lind, M. et al. Continuous glucose monitoring vs. conventional therapy for glycemic control in adults with type 1 diabetes treated with multiple daily insulin injections: the GOLD randomized clinical trial. JAMA 317, 379-387 (2017).

55. Gupta, S. et al. Factors associated with death in critically ill patients with coronavirus disease 2019 in the US. JAMA Intern. Med. https://doi. org/10.1001/jamainternmed.2020.3596 (2020).

56. Richardson, S. et al. Presenting characteristics, comorbidities, and outcomes among 5,700 patients hospitalized with COVID-19 in the New York City area. JAMA 323, 2052-2059 (2020).

57. Fiorentino, T. V. et al. Exenatide regulates pancreatic islet integrity and insulin sensitivity in the nonhuman primate baboon Papio hamadryas. JCI Insight https://doi.org/10.1172/jci.insight.93091 (2019).

58. Daniele, G. et al. The inflammatory status score including IL-6, TNF- $\alpha$, osteopontin, fractalkine, MCP-1 and adiponectin underlies whole-body insulin resistance and hyperglycemia in type 2 diabetes mellitus. Acta Diabetol. 51, 123-131 (2014).

59. Roder, M. E. et al. Intact proinsulin and beta-cell function in lean and obese subjects with and without type 2 diabetes. Diabetes Care 22, 609-614 (1999).

60. Matthews, D. R. et al. Homeostasis model assessment: insulin resistance and beta-cell function from fasting plasma glucose and insulin concentrations in man. Diabetologia 28, 412-419 (1985).

61. Song, Y. et al. Insulin sensitivity and insulin secretion determined by homeostasis model assessment and risk of diabetes in a multiethnic cohort of women: the Women's Health Initiative Observational Study. Diabetes Care 30 1747-1752 (2007).

62. Brandle, M., Lehmann, R., Maly, F. E., Schmid, C. \& Spinas, G. A. Diminished insulin secretory response to glucose but normal insulin and glucagon secretory responses to arginine in a family with maternally inherited diabetes and deafness caused by mitochondrial tRNA(LEU(UUR)) gene mutation. Diabetes Care 24, 1253-1258 (2001). 


\section{Acknowledgements}

We thank the Fondazione Romeo and Enrica Invernizzi for outstanding support. P.F. and F.D. are supported by the Italian Ministry of Health grant RF-2016-02362512. F.D. is supported by a Società Italiana di Diabetologia (SID) Lombardia Grant and by the EFSD/JDRF/Lilly Programme on Type 1 Diabetes Research 2019. R.A. is supported by K24 AI116925. V.U. is supported by the Fondazione Diabete Ricerca (FO.DI.RI) SID fellowship.

\section{Author contributions}

L.M., M.B.N. and F.D. designed and performed experiments, analysed data, wrote and edited the paper; C.L., A.A., A.M., E.A., V.U. and A.J.S. designed and performed research, analysed the data and edited the paper; A.R., I.P., G.D., M.D.A., E.I., R.M.F., E.C., P.M., M.E.L., A.M.B., S.R. and A.R. collected and analysed data; G.F., D.C. and M.N. assisted with sample collection, pathology and analysis of data; P.S., R.A., J.V.B., F.F., G.V.Z. and M.G. coordinated research; P.F. conceived the study, designed research and wrote and edited the paper. All authors reviewed and edited the paper.

\section{Competing interests}

The authors declare no competing interests.

\section{Additional information}

Extended data is available for this paper at https://doi.org/10.1038/s42255-021-00407-6.

Supplementary information The online version contains supplementary material available at https://doi.org/10.1038/s42255-021-00407-6.

Correspondence and requests for materials should be addressed to P.F.

Peer review information Nature Metabolism thanks Baki Billah, Joel Dave and the other anonymous, reviewer(s) for their contribution to the peer review of this work. Primary Handling Editor: Christoph Schmitt.

Reprints and permissions information is available at www.nature.com/reprints.

Publisher's note Springer Nature remains neutral with regard to jurisdictional claims in published maps and institutional affiliations.

(C) The Author(s), under exclusive licence to Springer Nature Limited 2021 

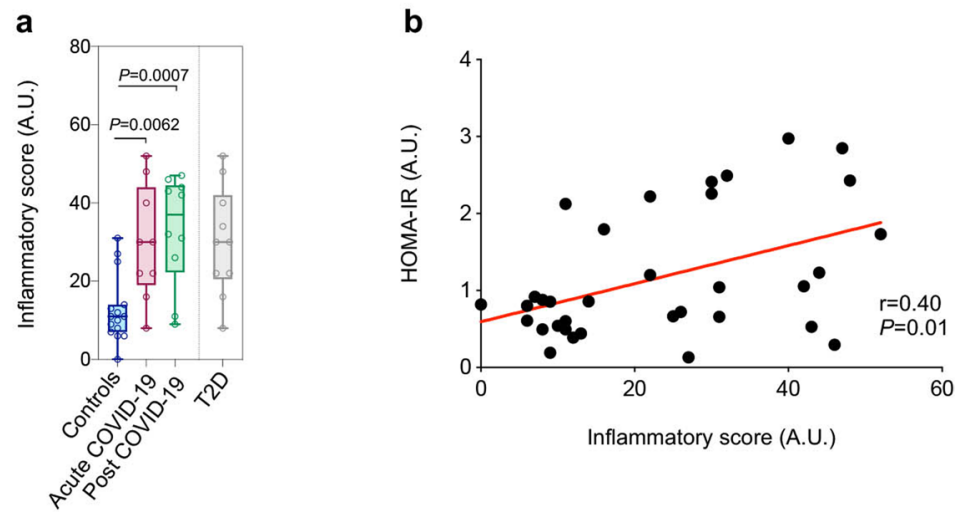

Extended Data Fig. 1 | Insulin resistance and high inflammatory score are evident in patients with COVID-19. a, Inflammatory score assessed in patients with COVID-19 (Acute COVID-19), in patients who recovered from COVID-19 (Post COVID-19), in patients with T2D and in healthy controls.

b, Correlation between HOMA-IR and inflammatory score (IS) in patients with COVID-19. Spearman's correlation analysis was performed. Red line reflects the best linear fit relationship between these variables (IS and HOMA-IR) with $p$ and $r$ represent two-sided Spearman rank-correlation tests. Data in

(a) are expressed as boxes and whiskers where the upper and lower bounds of the boxes represent the interquartile ranges. The horizontal line inside each box reflect the median and the whiskers indicate minimum and maximum values. Each dot represents an individual sample (Controls (blue), COVID-19 (maroon) and post-COVID-19 (moss)). Ordinary one-way ANOVA test with Bonferroni correction was used when applicable for calculating statistical significance between all groups. Data in (a) are representative of $n=15$ samples analyzed for controls, $n=9$ for Acute COVID-19, $n=10$ for Long COVID-19 and $n=10$ for patients with T2D. T2D group (shown in grey) is included for visual comparison only, ie it was not included in the statistical analysis though. 

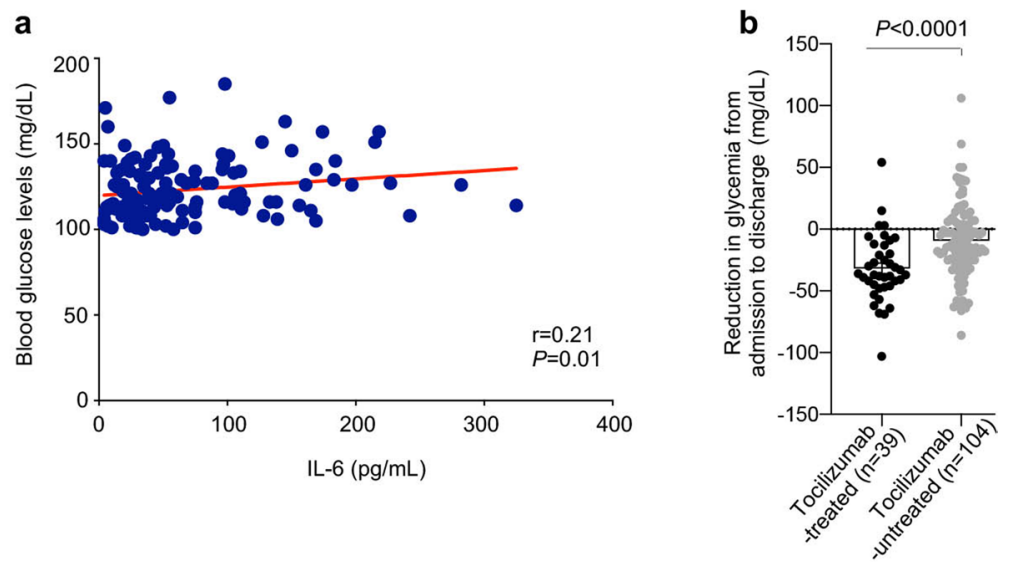

Extended Data Fig. 2 | Correlation between IL-6 and glucose and effect of tocilizumab on the latter in newly hyperglycemic patients with COVID-19.

a, Correlation between blood glucose and peripheral IL-6 levels in new-onset hyperglycemic patients with COVID-19 and (b) Reduction in glycemic levels expressed as delta between admission vs. discharge in new-onset hyperglycemic patients with COVID-19 and those treated or untreated with Tocilizumab. Red line shown in (a) reflects the best linear fit relationship between these variables (IL-6 levels and Blood glucose levels) with $p$ and $r$ represent two-sided Spearman rank-correlation tests. Data in (b) are represented as scatter dot plots showing the mean \pm SEM. Each dot represents an individual sample (COVID-19 treated with Tocilizumab (black), and COVID-19 untreated with Tocilizumab (grey)). Statistical significance was determined by unpaired $t$ test with welch correction. 


\section{Reporting Summary}

Nature Research wishes to improve the reproducibility of the work that we publish. This form provides structure for consistency and transparency in reporting. For further information on Nature Research policies, see our Editorial Policies and the Editorial Policy Checklist.

\section{Statistics}

For all statistical analyses, confirm that the following items are present in the figure legend, table legend, main text, or Methods section.

n/a Confirmed

$\square \bigotimes$ The exact sample size $(n)$ for each experimental group/condition, given as a discrete number and unit of measurement

$\square$ \ A statement on whether measurements were taken from distinct samples or whether the same sample was measured repeatedly

$\square$ The statistical test(s) used AND whether they are one- or two-sided

$\square$ Only common tests should be described solely by name; describe more complex techniques in the Methods section.

$\bigotimes \square$ A description of all covariates tested

$\square$ \A description of any assumptions or corrections, such as tests of normality and adjustment for multiple comparisons

$\square$ A full description of the statistical parameters including central tendency (e.g. means) or other basic estimates (e.g. regression coefficient)

$\triangle$ AND variation (e.g. standard deviation) or associated estimates of uncertainty (e.g. confidence intervals)

$\square$ For null hypothesis testing, the test statistic (e.g. $F, t, r$ ) with confidence intervals, effect sizes, degrees of freedom and $P$ value noted

Give $P$ values as exact values whenever suitable.

Х $\square$ For Bayesian analysis, information on the choice of priors and Markov chain Monte Carlo settings

Х $\square$ For hierarchical and complex designs, identification of the appropriate level for tests and full reporting of outcomes

Х $\square$ Estimates of effect sizes (e.g. Cohen's $d$, Pearson's $r$ ), indicating how they were calculated

Our web collection on statistics for biologists contains articles on many of the points above.

\section{Software and code}

Policy information about availability of computer code

Data collection all softwares used to perform data collection are described in the method section of the manuscript.

Data analysis we used the following softwares:

1- Stata version 12

2- GraphPad Prism version 8.4.3

3- Microsoft Excel version 16.30

For manuscripts utilizing custom algorithms or software that are central to the research but not yet described in published literature, software must be made available to editors and reviewers. We strongly encourage code deposition in a community repository (e.g. GitHub). See the Nature Research guidelines for submitting code \& software for further information.

\section{Data}

Policy information about availability of data

All manuscripts must include a data availability statement. This statement should provide the following information, where applicable:

- Accession codes, unique identifiers, or web links for publicly available datasets

- A list of figures that have associated raw data

- A description of any restrictions on data availability

all data generated or analyzed during this study are included in this submitted article and its supplementary information file. we have also included the following statement in our manuscript regarding data availability:

Data Availability

The data that support the findings of this study are available from the corresponding author upon request. Source data files are provided with this paper. 


\section{Field-specific reporting}

Please select the one below that is the best fit for your research. If you are not sure, read the appropriate sections before making your selection. $\bigotimes$ Life sciences $\quad \square$ Behavioural \& social sciences $\quad \square$ Ecological, evolutionary \& environmental sciences

For a reference copy of the document with all sections, see nature.com/documents/nr-reporting-summary-flat.pdf

\section{Life sciences study design}

All studies must disclose on these points even when the disclosure is negative.

Sample size sample size was set as 15 in control group and 10 in other subgroups thus allowing for $80 \%$ power to detect at least $15 \%$ of difference between all groups with a significance level of $\mathrm{a}=0.05$ as reported in the method section and according the following publications:

1- Brandle, M., Lehmann, R., Maly, F. E., Schmid, C. \& Spinas, G. A. Diminished insulin secretory response to glucose but normal insulin and glucagon secretory responses to arginine in a family with maternally inherited diabetes and deafness caused by mitochondrial tRNA(LEU(UUR)) gene mutation. Diabetes Care 24, 1253-1258, doi:10.2337/diacare.24.7.1253 (2001).

2- Solerte, S. B. et al. Sitagliptin Treatment at the Time of Hospitalization Was Associated With Reduced Mortality in Patients With Type 2 Diabetes and COVID-19: A Multicenter, Case-Control, Retrospective, Observational Study. Diabetes Care, doi:10.2337/dc20-1521 (2020).

Data exclusions no data were excluded from the analysis.

Replication The experiments were done on 2 subgroups and were successfully replicated on the control group.

Randomization Allocation of samples/subjects was random.

Blinding Data analysis was not blinded since the experiment was based on quantitative measurements.

\section{Reporting for specific materials, systems and methods}

We require information from authors about some types of materials, experimental systems and methods used in many studies. Here, indicate whether each material, system or method listed is relevant to your study. If you are not sure if a list item applies to your research, read the appropriate section before selecting a response.

Materials \& experimental systems

$\mathrm{n} / \mathrm{a}$ Involved in the study

Х $\square$ Antibodies

\ $\square$ Eukaryotic cell lines

\ $\square$ Palaeontology and archaeology

\ $\square$ Animals and other organisms

$\square$ \uman research participants

\ $\square$ Clinical data

$\bigotimes \square$ Dual use research of concern

\section{Human research participants}

Policy information about studies involving human research participants

Population characteristics

A detailed description of clinical, demographic characteristics of the study participants are provided in the method section of the manuscript and in Tables 1, 2 and S1.

Recruitment

Ethics oversight

\begin{tabular}{l|l}
\multicolumn{2}{l}{ Methods } \\
\hline n/a & Involved in the study \\
$\searrow$ & $\square$ ChIP-seq \\
$\square$ & $\square$ Flow cytometry \\
$\square$ & $\square$ MRI-based neuroimaging
\end{tabular}

Controls selection was based on the recruitment of healthy-conscious individuals who have a negative test for COVID-19 and a negative clinic as per evaluation of the Infectious Disease and Respiratory Division of ASST FBF-Sacco Milan. Those controls have no previous history for diabetes, thus no bias selection was evident in our scientific conclusions. Similar methodology was used for recruiting patients with COVID-19 and those who recovered from COVID-19 following the aforementioned guidelines as stated in details in our method section.

The ethical approval was granted by Local Ethical Research Committee of Milan (Comitato Etico Milano Area 1), which was already mentioned in the method section of our manuscript and here is the full address: c/o ASST FBF Sacco - P.O. L. Sacco, Via G.B. Grassi n. 74, 20157 Milano

tel. 02 3904.3518/3522/2094

mail: comitato.etico@asst-fbf-sacco.it 